\title{
Computerization of Clinical Records in Out-Patient Departments of Sri Lankan Hospitals
}

\author{
Dr. D. Pole MBBS, MD \\ Independent Consultant in Medical Informatics \\ E-mail :drpole@gmail.com
}

Sri Lanka Journal of Bio-Medical informatics 2010;1(4):200-04

DOI: $10.4038 /$ sljbmi.v1i4.2235

\begin{abstract}
Electronic Patient Records (ePR) have been implemented in 27 government hospitals in the Eastern Province of Sri Lanka in a project funded by the Austrian/Swiss Red Cross that ran from 2006 to 2009. The computers store a summary of the clinical record, which is easily accessible when the patient returns. They are also used to print various reports such as discharge letters, prepare quarterly statistics of the diseases treated and notify infectious diseases.

In the original design of this Multi-Disease Surveillance project, it was planned to use computers mainly for recording in-patient details. However as the project proceeded, it became clear that major gains in efficiency and quality of documentation could be made by using computers in the out-patient departments (OPD). Providing computers for the doctors to use in OPD has greatly increased the speed of documentation as well as providing access to the previous clinical record. Computer recording of prescriptions has streamlined the supply of drugs and stock control in the hospital pharmacy.
\end{abstract}

In some of the hospitals, a connection to the Internet allows staff to browse the latest medical knowledge related to the patient being treated.

Keywords: e-Health; Electronic Patient Record; Medical Informatics; Developing Countries; Multi-Disease Surveillance

\section{Introduction}

The Multi-Disease Surveillance project (MDS) was implemented by the Austrian/Swiss Red Cross Societies in 2006-2009 with tsunami rehabilitation funds of approximately 150 million LK Rupees. The software was developed by the project team in collaboration with WHO ${ }^{(1)}$. It runs under the Caché database system, a very efficient database engine developed by Intersystems Corporation initially for use in hospitals in the USA ${ }^{(2)}$. Systems were installed in 23 provincial and 4 line-ministry hospitals in the Eastern Province of Sri Lanka to store clinical information on patients.

While the initial plan was to concentrate on the capture and processing of admission data, it 
became clear during the project that the biggest gains in efficiency and improvement of the clinical documentation could be made by computerized record-keeping in the out-patient departments of the hospitals. It was there that documentation posed the biggest problems for the staff. Out-patient departments are heavily attended and the OPD staff so overloaded, that they could hardly find the time to write up adequate clinical documentation.

The system also helps staff to order and visualize laboratory tests and x-rays. In the latter case, the computer can display a digital $x$-ray image on the screen thus reducing the costs of $\mathrm{x}$-ray films. In those hospitals using computers for out-patient records, each OPD doctor has a screen on their desk and the electronic record replaces the use of paper. Administrative and statistical reports are produced on other computers connected to the network.

\section{Database design}

In the design of the MDS software it was necessary to strike a balance between enough information to make the stored patient record useful but not so complicated as to overwhelm the staff with too much detail. A general principle adopted was to duplicate as far as possible the layout of the current manual records and to resist the temptation to "improve" on them.

Figure 1. The out-patient record.

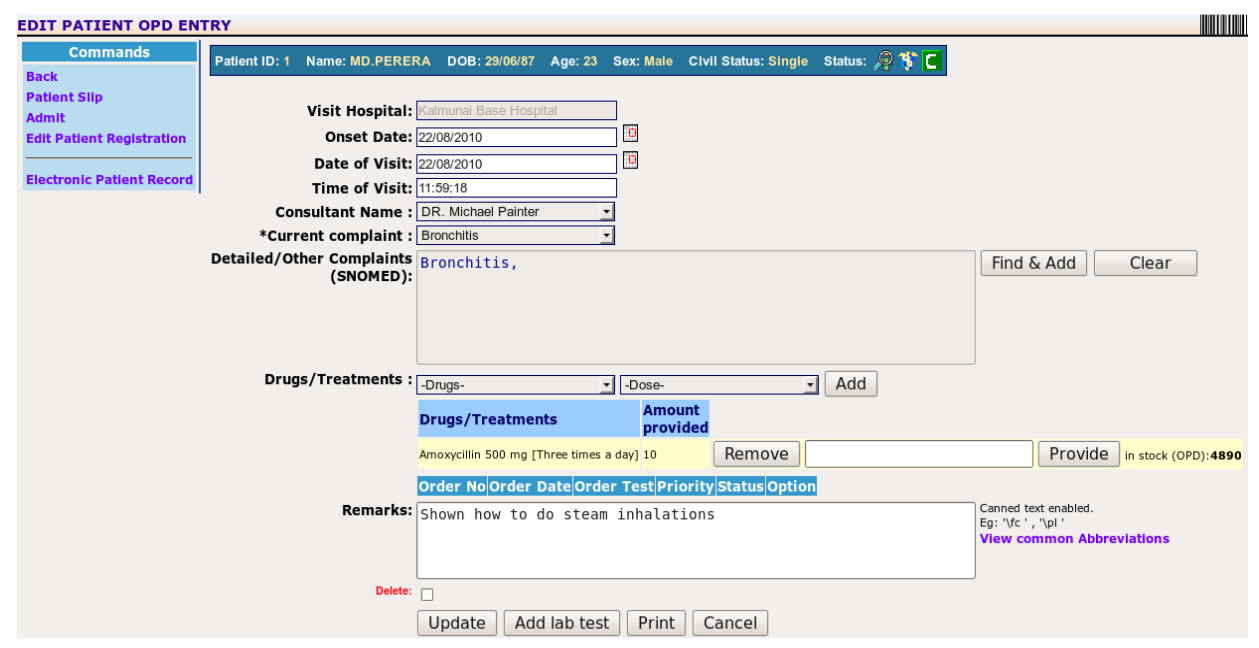

In one simple screen, all the information that needs to be recorded during a consultation is shown. The patient's personal details have already been recorded at the front desk when the patient sees the doctor and the rest of the information can be entered in less than 30 seconds (data and time of the visit, staff involved, patient complaint, treatment and lab tests). Items not coded can be entered as textual remarks if necessary. If necessary, a printed record can be produced for the patient. The same screen is seen by staff in the pharmacy where it is used to dispense the drugs prescribed. An important feature is that staff see the current stock of the medications to be supplied and can respond accordingly.

Only the patient's presenting complaint is obligatory; a doctor who wants to enter more details can do so but others can restrict themselves to the absolute minimum. In order to keep 
the input user-friendly, pop-up lists are used for simple choices like current complaint, while longer lists from official sources such as SNOMED terms are stored in the computer for easy browsing. The list of drugs available in the pharmacy and the tests carried out in the hospital's laboratory or x-ray department are also stored.

A problem was faced when codifying diseases. The ICD-10 coding, used by the health ministry for producing health statistics, is normally carried out by specially trained staff in the medical records department of each hospital. It was used in the first version of the software, as it is the health ministry's standard. However, its use proved to be too difficult for non statistically-trained hospital staff to use. SNOMED is much more clinically oriented for doctors and nurses to use and contains a link to ICD-10. SNOMED can therefore be used by the medical staff to enter a diagnosis (for example "Nucleotidase deficiency") and the computer finds the more administrative term used in the ICD-10 (in this case "Disorders of plasma-protein metabolism, not elsewhere classified").

However the main benefit perceived by the medical staff is access to the previous medical record. On one screen, it is possible to see in overview the total record of the patient's attendances to the hospital - OPD consultations, Clinic visits and Admissions. Individual records can then be opened to show more detail:

Figure 2. Overview of the entire medical record

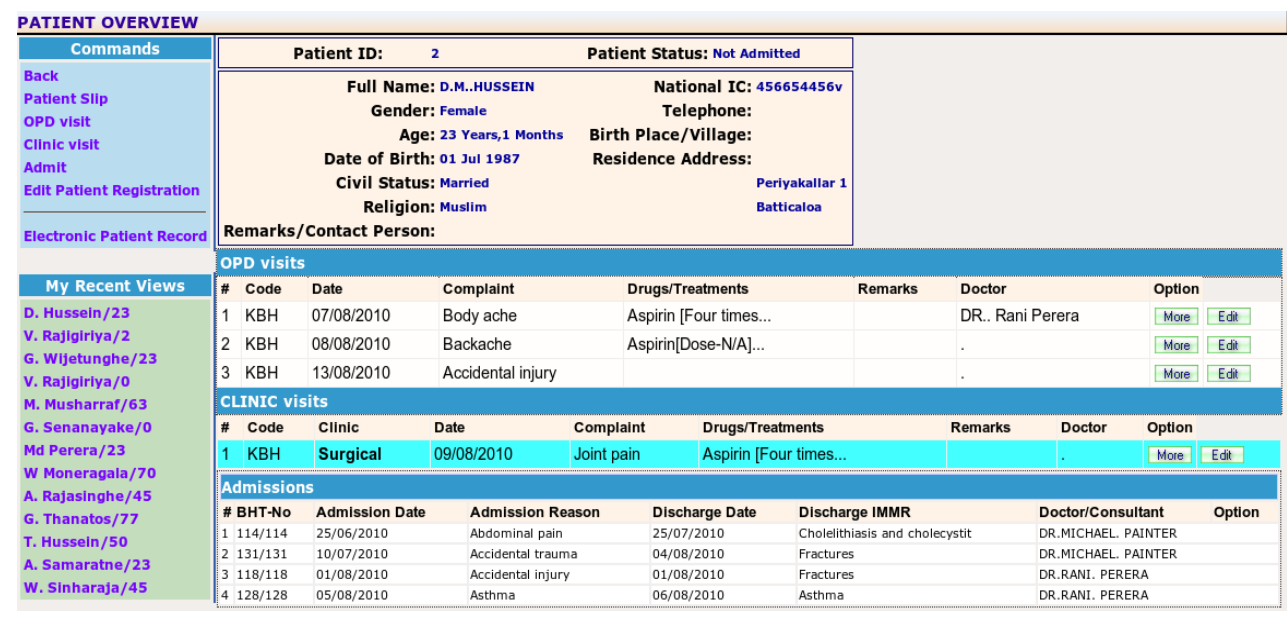

As well as keeping the records of individual patients, the computer provides the hospital director with useful information to help run the hospital. This covers hospital performance statistics, patient listings, drugs available and those routinely prescribed, personnel information such as holiday leave and pay increments and so on. The system contributes to public health by alerting the staff when there is a communicable disease that must be notified. It also produces quarterly health statistics automatically. A module that maps the notifiable diseases and provides daily statistics on the complaints and treatment provided for outpatients is currently being tested. 


\section{Results}

By the end of the project, systems had been installed in a total of 27 hospitals and maintenance of the systems in 23 of these hospitals taken over by the Provincial Health Service. Successful use of the systems admitted patients was measured by a) recording the admission and discharge of all patients admitted, b) stopping of manual recording in the admission book and c) computerized production of the Inpatient Morbidity and Mortality Return (IMMR) at the end of each quarter. In the case of out-patient use, success was measured by the recording of all OPD visits in the computer at the time of the patient's visit.

On these criteria, at the end of the project admissions were being successfully covered in $50 \%$ of hospitals and OPD visits in $11 \%$. Six months later (July 2010), the implementation rates had risen to $70 \%$ coverage for admissions and $18 \%$ for OPD visits. These measurements were made by the implementation team however, and may be subject to bias. In future, the results will be measured by government IT staff to ensure greater objectivity.

\section{Discussion}

While the health services of developing countries like Sri Lanka obviously face many problems, computerization can transform many of these into opportunities. It is important however, to avoid cost and to resist complexity. The model used in this project was for a separate local area network in each hospital with connection to other institutions if necessary via Internet. Hardware and development costs were kept down by using the highly efficient, easy to program Caché database and the LINUX operating system. This combination provided excellent performance on inexpensive computers, and web-based programming made it easy to maintain the software from a central location.

The key to success however was training. During the 4 years of the implementation, over 1000 staff were trained and the Red Cross team provided continuous user support. It took most hospitals about one year before their skills were up to the level of running the systems by themselves, but outside support and regular training will always be necessary to ensure that the systems continue to function and that new staff are able to use the computer.

The main source of statistical data on disease and death in Sri Lanka has been for many years the "Indoor Morbidity and Mortality Return" (IMMR) from each government hospital. This return, collected quarterly, is prepared by medical record staff in each government hospital who codify the discharge diagnosis written by doctors into the ICD-10 classification. However, this return only covers admitted patients. As their records are coded some time after the patient has been discharged, it may not be fully accurate. In fact, a study of bed-head tickets several years ago $^{(3)}$ showed that the diagnosis was not always recorded in the admission notes and was sometimes inaccurate. As the coding is done by the computer at the time of discharge, it is expected that it will be more accurate.

Having OPD data stored directly into the computer will allow hospitals to provide regular 
statistical reports on the diseases treated in out-patients.

\section{Conclusions}

Data will only be complete if the computer systems are fully integrated into the day-to-day activities of each hospital; at the patient reception and examination areas and in every place where clinical data is recorded. Data recording must also be easier than the manual method and the computer software must be fast and user-friendly for its use to become general.

As computer systems start to replace manually written records, it is vitally important to maintain them in order to guarantee their continuous operation. Computer hardware and peripherals need to be regularly checked and faulty equipment repaired. The local area networks need to be maintained and extended when necessary and the functionality of the software must be regularly kept up to date. As well as this preventive maintenance, a rapid response to system failure (Hotline Service) is necessary.

Now that the MDS implementation period is over, maintenance of the systems in the 23 provincial hospitals of the project has been taken over by the provincial health authorities. There are regular visits to each hospital by technical experts, who provide hardware and software support and training. Currently, one year after the Red Cross project finished, systems in all the 23 provincial hospitals in the project are running and being used.

\section{References}

1. WHO (2004): "Comprehensive Assessment of National Surveillance Systems in Sri Lanka ", WHO - SEARO, February 2004

2. Intersystems [Online]. [Cited 2010 Nov 22];

Available from URL: http:// www.intersystems.com

3. Uni. Colombo (2002) "MSc Dissertation", Dr S. Gamage, NIHS, University of Colombo 2002 